\title{
Jitter Equalization for Binary Baseband Communication
}

\author{
Anthony Chan Carusone \\ Department of Electrical and Computer Engineering \\ University of Toronto \\ Toronto, Canada \\ Email: tcc@eecg.utoronto.ca
}

\begin{abstract}
A new adaptation strategy for the equalization of high speed binary links is presented which reduces patterndependent jitter. Instead of using the LMS or a related algorithm that optimizes the vertical eye opening at the receiver, the equalizer can be optimized to reduce intersymbol interference at data transitions, thereby targeting zero jitter. The LMS algorithm is modified to combine the two criteria and provide a trade off between vertical eye opening and horizontal eye opening at the equalizer output. Behavioral simulations verify the technique on a lossy transmission line model.
\end{abstract}

\section{INTRODUCTION}

An equalizer mitigates the effects of intersymbol interference (ISI) in bandlimited communication systems. In binary baseband links, equalizers are usually used to improve the noise margin (vertical eye opening) at a receiver. However, in high speed (multi-Gb/s) links, timing margin (horizontal eye opening) is often a more critical system parameter. ISI at transitions in the received waveform result in patterndependent jitter that contributes significantly to the total jitter at the receiver [1]. In high speed links with little timing margin, an equalizer that minimizes the pattern-dependent jitter may be preferable to one optimized for minimal intersymbol interference at the center of the eye.

This paper introduces "jitter equalization". First, in Section II, a criteria for zero pattern-dependent jitter over a binary linear channel is reviewed. Then, adaptation algorithms that target this condition are developed in Section III. Their performance is compared with the traditional LMS algorithms common in current high speed binary links via behavioral simulations.

\section{ZERO JITTER CRITERION}

Consider a channel with pulse response $h(t)$ communicating binary data at a baud rate of $T$. Without loss of generality, assume that the pulse response is roughly centered around time $t=0$. The familiar Nyquist 1 criterion guarantees zero intersymbol interference at all sampling instants.

$$
h(k T)= \begin{cases}1, & k=0 \\ 0, & k \neq 0\end{cases}
$$

However, the channel output at the transition times, $u(k T+$ $T / 2$ ), depends upon different samples of the pulse response.

$$
u(k T+T / 2)=\sum_{l=-\infty}^{\infty} h((k+l) T+T / 2) d(k-1)
$$

To ensure that $u(k T+T / 2)=0$ for any transmitted data pattern $d(k)= \pm 1$, implying zero jitter at transitions of the received waveform, the channel's pulse response must satisfy the Nyquist 2 criterion:

$$
h\left(k T+\frac{T}{2}\right)= \begin{cases}h\left(\frac{T}{2}\right), & k=-1 \\ 0, & k \neq-1,0\end{cases}
$$

Pulses which satisfy the Nyquist 1 criterion (1) and Nyquist 2 criterion (3) are shown in Figure 1 (a) and (b) respectively for comparison. Note that pulses satisfying only the Nyquist 1 criterion may still have considerable intersymbol interference at the transition times and, hence, a lot of pattern-dependent jitter.

Of course, if the channel itself does not satisfy the Nyquist 2 criterion, it is possible to introduce a linear filter at either the transmitter or receiver so that the pulse response of the cascaded system comes closer to satisfying (3). This will be referred to as "jitter equalization".

\section{AdAPTIVE JITTER EQUALIZATION}

In this section, an adaptive filter is used to minimize the jitter in a binary baseband receiver. Figure 2 shows a block diagram of a system with adaptive equalization at the receiver. Although a linear equalizer may equivalently be placed at the transmitter, adaptation would be complicated by the need to somehow relay information from the receiver back to the transmitter.

Most popular techniques for adaptive equalization in hardware strive to minimize the mean squared error in the output of the equalizer at the center of the received eye.

$$
\xi=\mathrm{E}\left[(r(k T)-d(k))^{2}\right]
$$

In (4), $r(t)$ is the output of the equalizer, $d(k)$ is the transmitted binary sequence (hence, the desired equalizer output), and $\mathrm{E}[\cdot]$ denotes expectation. This approach effectively maximizes the vertical eye opening of the received data at the sampling instant only.

If jitter is of primary concern, it is preferable to minimize the mean squared error at transitions in the received binary waveform.

$$
\zeta=\mathrm{E}\left[\left.r\left(k T+\frac{T}{2}\right)^{2}\right|_{d(k+1) \neq d(k)}\right]
$$




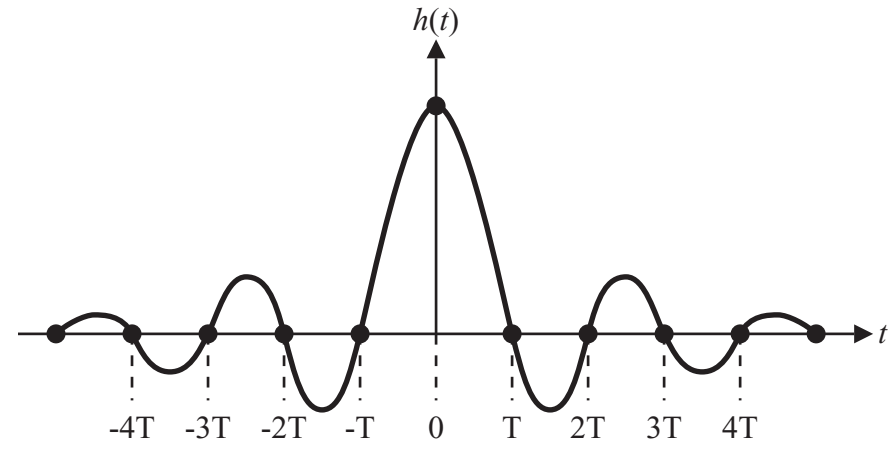

(a)

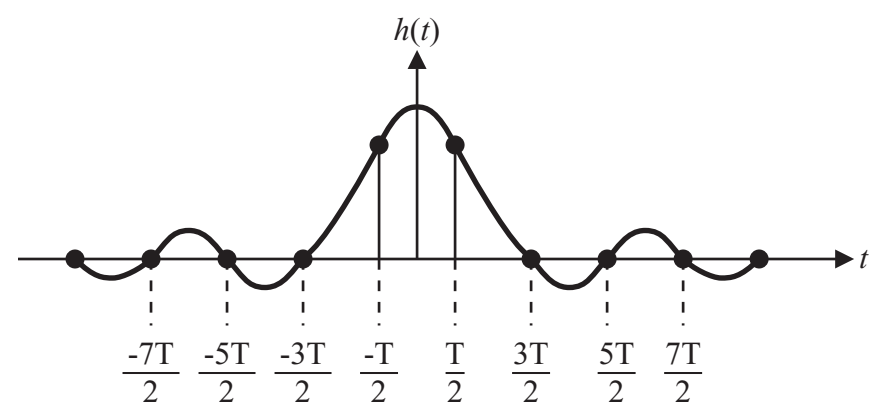

(b)

Fig. 1. (a) A pulse satisfying the Nyquist criteria for zero intersymbol interference. (b) A pulse satisfying the criteria for zero jitter when transmitting binary data.

In (5), only transitions in the received binary waveform are included in the expectation operator. (i.e. Samples halfway between consecutive +1 's or -1 's are not included since they do not contribute to zero crossing jitter.) The difference between $\xi$ and $\zeta$ is illustrated in Figure 3, which plots the unequalized eye diagram at the end of a channel modeled by the pulse response in Figure 4. Note that in the absence of noise, when $\zeta=0$ all zero crossings are equally spaced and the zero jitter criteria expressed in (3) is satisfied by the pulse response of the channel and equalizer combined.

The least mean square (LMS) algorithm, commonly employed for the adaptation of equalizers, updates filter parameters iteratively based upon sampled values of the output error.

$$
p_{i}(k T)=p_{i}\left(k T-\frac{T}{2}\right)+2 \mu_{1} u(k T-i T)(r(k T)-d(k))
$$

In (6), $u(t)$ is the received waveform at the input to the equalizer.

It can be shown that the LMS algorithm minimizes $\xi$. However, it is easily modified to minimize $\zeta$ by using the value of the received waveform at data transitions as the error signal. When there are no transitions in the received data (i.e. two consecutive +1 's or -1 's) the adaptation should wait until a transition is received. The resulting iterative update rule is described by equation (7) at the top of the page.

Unfortunately, a trivial solution to minimizing $\zeta$ is to set

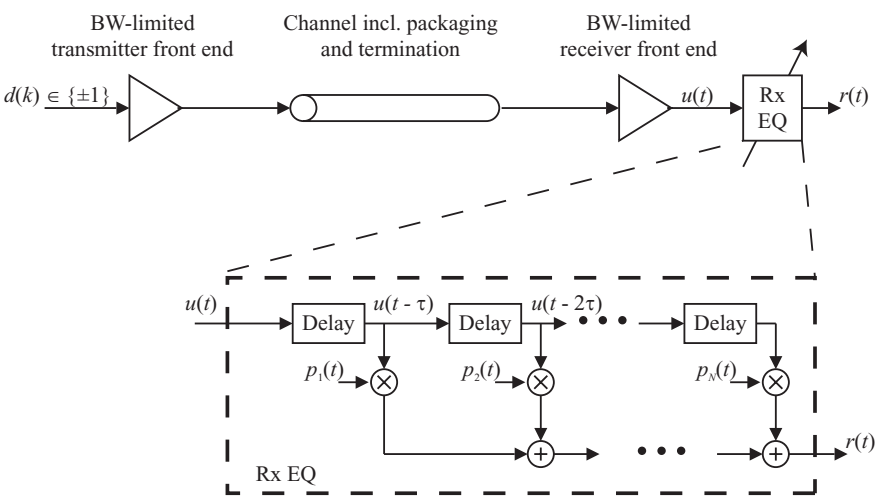

Fig. 2. The system model used for behavioral simulations.

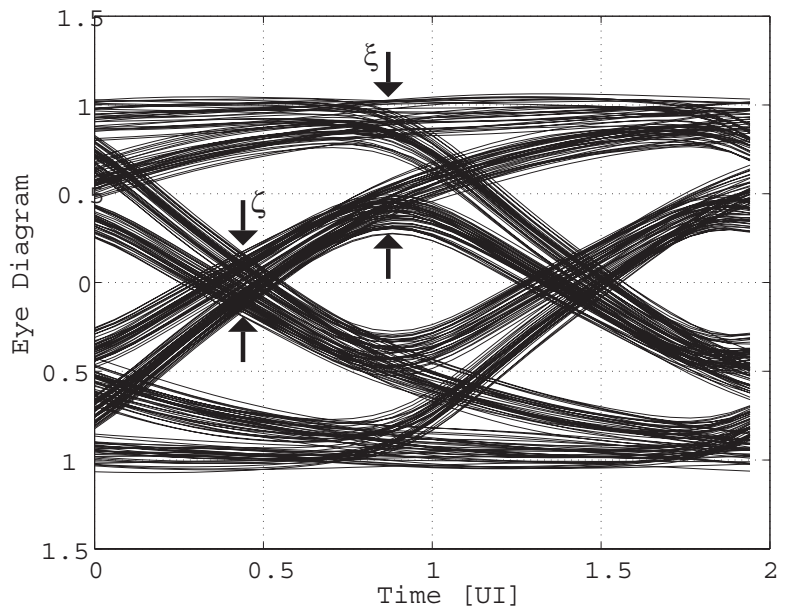

Fig. 3. Different mean squared error criteria for adapting an equalizer for binary data. The eye diagram is taken at the end of an unequalized channel modeled by the pulse response in Figure 4

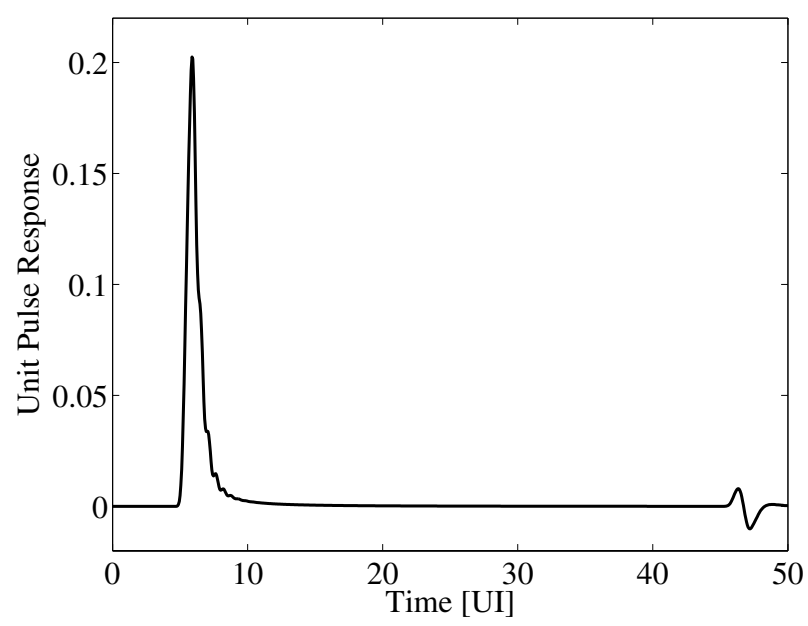

Fig. 4. Pulse response for behavioral simulations derived from a lossy transmission line model with mismatched terminations and bandwidth limited transmitter and receiver. 


$$
p_{i}\left(k T+\frac{T}{2}\right)= \begin{cases}p_{i}(k T)+2 \mu_{2} u\left(k T+\frac{T}{2}-i T\right) r\left(k T+\frac{T}{2}\right), & d(k+1) \neq d(k) \\ p_{i}(k T), & d(k+1)=d(k)\end{cases}
$$

TABLE I

COMPARING THE PERFORMANCE OF JITTER EQUALIZATION WITH A TRADITIONAL LMS EQUALIZER. THE RESULTS ARE WORST-CASE BASED ON A PEAK DISTORTION ANALYSIS [2].

\begin{tabular}{|l|c|c|}
\hline & $\begin{array}{c}\text { Normalized vertical } \\
\text { eye opening }\end{array}$ & $\begin{array}{c}\text { Peak-to-Peak Pattern } \\
\text { Dependent Jitter [UI] }\end{array}$ \\
\hline No eq. & 0.2000 & 0.41 \\
\hline LMS eq. & 0.8260 & 0.22 \\
\hline Jitter eq. & 0.7788 & 0.17 \\
\hline
\end{tabular}

all of the equalizer parameters, $p_{i}$, equal to zero. That is, to turn the equalizer off. Without special care the adaptation algorithm will converge to this trivial condition, clearly not the intended result. This can be avoided by fixing the value of one "main" tap weight. However, experimentation indicated that it is preferable to combine traditional LMS adaptation and jitter-minimizing adaptation by alternately iterating equations (6) and (7). The result is a compromise between maximizing the vertical and horizontal eye openings. Scaling the values of the adaptation constants, $\mu_{1}$ and $\mu_{2}$, one can control the tradeoff between noise margin and timing margin at the equalizer output. For the central "main" tap weight, only LMS adaptation (6) is used to ensure a constant nominal eye opening.

Simulations were performed using PRBS $2^{31}-1$ binary data transmitted over the channel model in Figure 4, which is derived from a lossy transmission line model with mismatched terminations and a bandwidth limited transmitter and receiver. An ideal 7 tap FIR filter with $T / 2$-spaced taps is used for equalization. Both the traditional LMS algorithm and the compromise jitter equalization technique described above were used to optimize the tap weights. Convergence of the tap weights is plotted over time in Figure 5 and the received eye diagrams after convergence are plotted in Figure 6.

Notice that the traditional LMS algorithm efficiently cancels ISI at the center of the eye providing excellent noise margin at the sampling instant. However, there is significant zerocrossing jitter. Adapting the same equalizer using the compromise jitter equalization strategy provides approximately $6 \%$ less vertical eye opening in exchange for approximately $25 \%$ less pattern-dependent jitter. The results are summarized in Table I.

The pulse responses of the channel/equalizer combination after convergence are plotted in Figure 7. Baud-rate samples contributing to the centre of the eye are identified by circles whereas samples of the impulse response contributing to jitter are identified with crosses. It is instructive to compare these results with the idealized pulse responses in Figure 1.

Using the traditional LMS algorithm, (Figure 7(a)), the

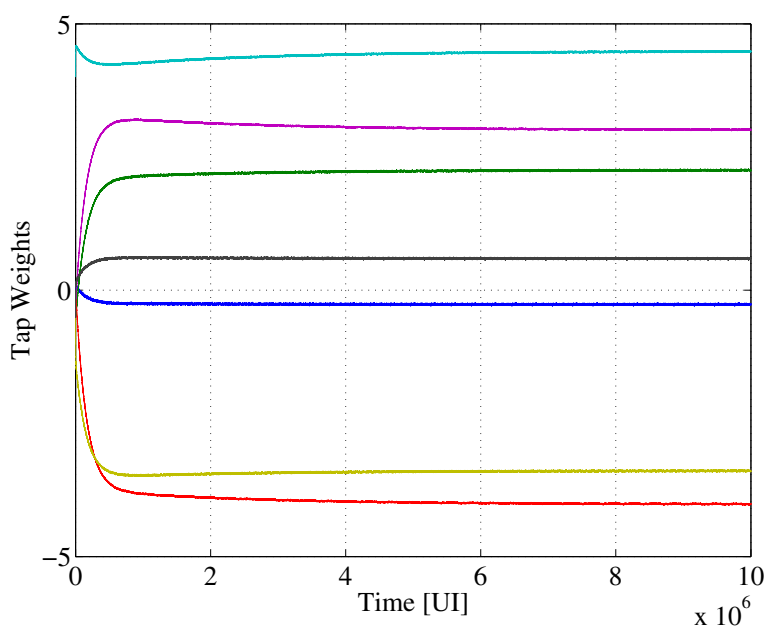

(a)

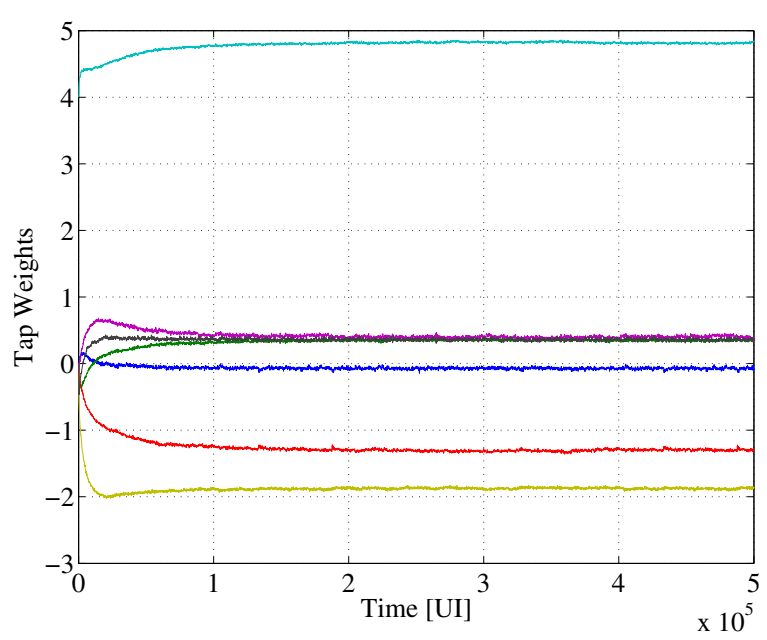

(b)

Fig. 5. Tap weight adaptation over time using (a) LMS adaptation, and (b) Jitter equalization.

baud-rate samples identified by circles nearly satisfy the Nyquist 1 criterion, (1). However, a significant pre-cursor pulse is apparent. Since the pulse is not aligned with the centre of the eye, it is not canceled by the adaptive equalizer which strives only to minimize $\xi$. As a result, the baud-rate samples identified by crosses do not nearly satisfy the Nyquist 2 criterion (3), and the pre-cursor pulse causes significant pattern-dependent jitter.

On the other hand, the pulse response obtained using jitter equalization plotted in Figure 7(b) has no major pre- or postcursor ISI. It nearly satisfies both the Nyquist 1 and 2 criteria. 


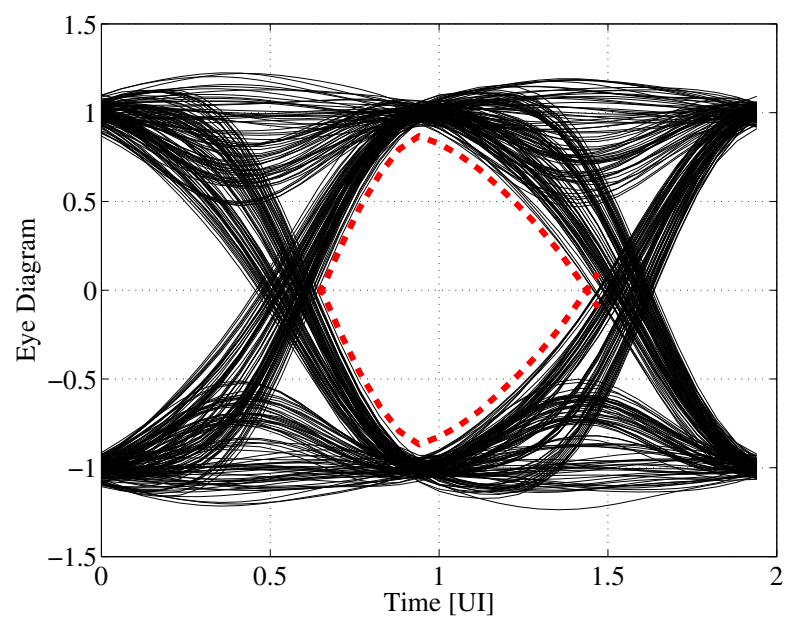

(a)

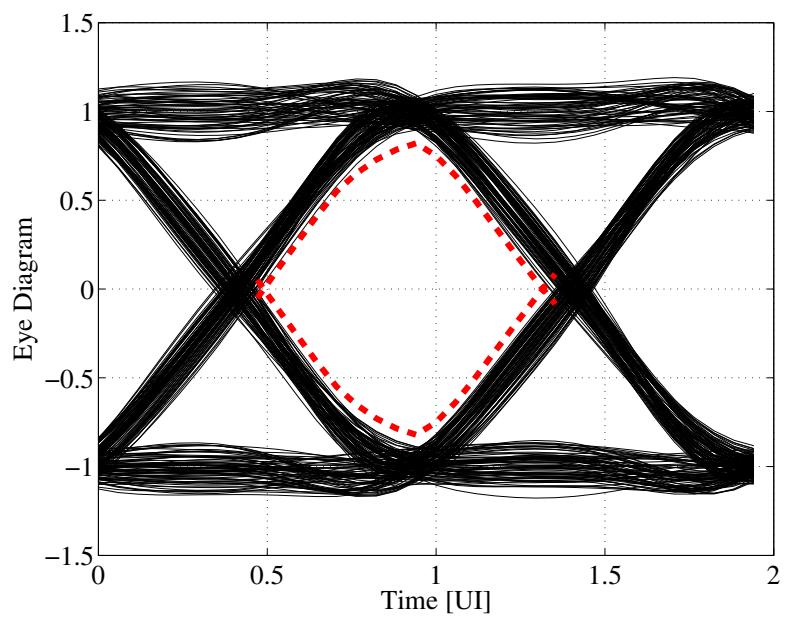

(b)

Fig. 6. Equalized eye diagrams for PRBS $2^{31}-1$ binary data over an ISI channel using (a) LMS adaptation, and (b) Jitter equalization.

\section{CONCLUSION}

For high speed (multi Gb/s) binary communication links, timing margin at the receiver is often more critical than noise margin. Pattern-dependent jitter decreases timing margin at the receiver and hinders the recovery of a low-jitter clock. However, bandwidth limited channels are generally equalized using the LMS or related algorithms which are designed to optimize the vertical eye opening. Instead, if zero jitter is targeted by the adaptation algorithm, pattern-dependent jitter can be greatly reduced while still increasing the vertical eye opening at the receiver (although somewhat less than using traditional LMS adaptation). A technique has been described to combine the two optimization criteria thereby providing a trade off between vertical eye opening and horizontal eye opening. This allows one to strike a balance between the sensitivity and timing requirements of a receiver. Behavioral simulations verified the technique on a lossy transmission line model.

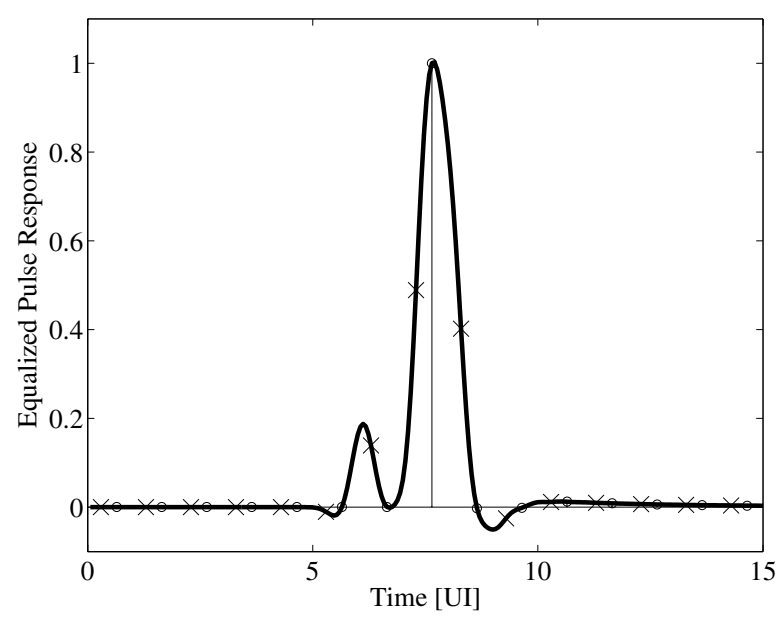

(a)

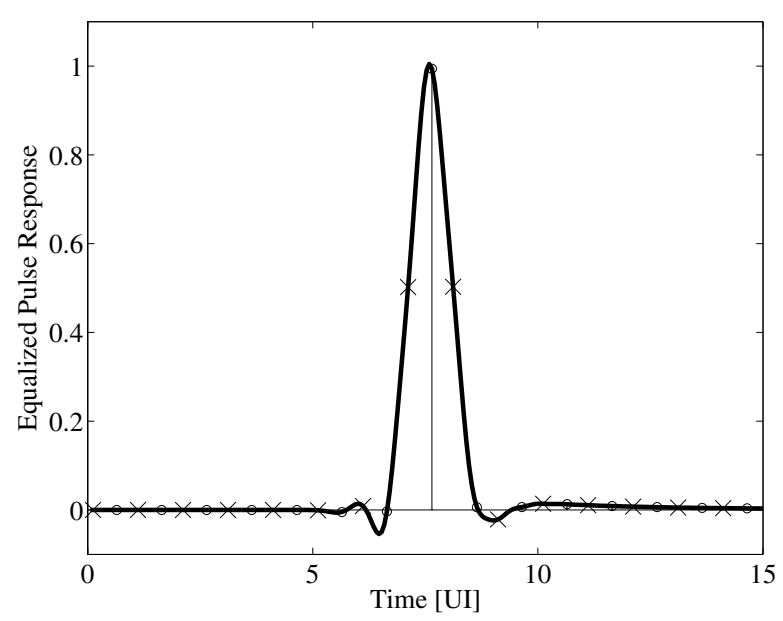

(b)

Fig. 7. Equalized pulse responses for the channel model in Figure 4 using (a) LMS adaptation, and (b) Jitter equalization. Circles denote samples contributing to the centre of the received eye while crosses identify samples contributing to zero crossing jitter.

The complexity of implementing the new iterative update rule (7) is comparable to the traditional LMS algorithm (6). However, a practical drawback of jitter equalization is that, unlike the LMS algorithm, it requires the received waveform to be sampled twice per baud interval: once at the centre of the eye and once at its transitions. Since sampling at the transition times is generally also required for phase detection and clock recovery, this may not imply an additional overhead in a highly integrated receiver.

\section{REFERENCES}

[1] V. Stojanovic and M. Horowitz, "Modeling and analysis of high-speed links," in Custom Integrated Circuits Conf., Sept. 2003, pp. 589-594.

[2] B. K. Casper, M. Haycock, and R. Mooney, "An accurate and efficient analysis method for Gb/s chip-to-chip signaling schemes," in Symp. VLSI Circuits, June 2002, pp. 54-57. 